\title{
Arithmetic properties arising from Ramanujan's theta functions
}

\author{
M. S. Mahadeva Naika ${ }^{1}$ • D. S. Gireesh ${ }^{1}$
}

Received: 27 May 2015 / Accepted: 4 November 2015

(C) Springer Science+Business Media New York 2016

\begin{abstract}
We prove some interesting arithmetic properties of theta function identities that are analogous to $q$-series identities obtained by Michael D. Hirschhorn. In addition, we find infinite family of congruences modulo powers of 2 for representations of a non-negative integer $n$ as $\triangle_{1}+4 \triangle_{2}$ and $\Delta+k \square$.
\end{abstract}

Keywords Theta functions - Arithmetic properties - Triangular numbers · Congruences

Mathematics Subject Classification $14 \mathrm{~K} 25 \cdot 11 \mathrm{P} 83$

\section{Introduction}

Let $a_{k}(n)$ be the number of representations of $n$ as $\triangle_{1}+2 k \triangle_{2}$ and $b_{k}(n)$ be the number of representations of $n$ as $\triangle+k \square$. Then

$$
\begin{aligned}
\sum_{n=0}^{\infty} a_{k}(n) q^{n} & =\psi(q) \psi\left(q^{2 k}\right), \\
\sum_{n=0}^{\infty} b_{k}(n) q^{n} & =\psi(q) \varphi\left(q^{k}\right) .
\end{aligned}
$$

This research was partially supported by DST Govt. of India under the Project Grant SR/S4/MS:739/11.

M. S. Mahadeva Naika msmnaika@rediffmail.com

D. S. Gireesh

gireeshdap@gmail.com

1 Department of Mathematics, Bangalore University, Central College Campus, Bangalore, Karnataka 560 001, India 
Hirschhorn and Sellers [3] used some interesting $q$-series identities to obtain results of congruences modulo 5 for 4-colored Frobenius partitions, and Hirschhorn [2] proved equivalent results of $q$-series identities that are used in [3]: If

$$
\sum_{n \geq 0} g(n) q^{n}=\frac{\left(q^{2} ; q^{2}\right)_{\infty}^{2}\left(q^{4} ; q^{4}\right)_{\infty}^{3}}{(q ; q)_{\infty}} \text { and } \sum_{n \geq 0} h(n) q^{n}=\frac{(q ; q)_{\infty}^{3}\left(q^{2} ; q^{2}\right)_{\infty}^{2}}{\left(q^{4} ; q^{4}\right)_{\infty}}
$$

where $(a ; q)_{\infty}=\prod_{n \geq 0}\left(1-a q^{n}\right)$, then

$$
\sum_{n \geq 0} g(5 n) q^{n}=\sum_{n \geq 0} h(n) q^{n}-5 \sum_{n \geq 0} g(n) q^{5 n+3}
$$

and

$$
\sum_{n \geq 0} h(5 n+3) q^{n}=16 \sum_{n \geq 0} g(n) q^{n}-5 \sum_{n \geq 0} h(n) q^{5 n} .
$$

Motivated by these works, in Sect. 3, we establish arithmetic properties of representations of a number $\Delta_{1}+2 k \Delta_{2}$ and $\Delta+k \square$. In addition, we also find certain congruences modulo powers of 2 for number of representations of a non-negative integer $n$ as $\triangle_{1}+4 \triangle_{2}$ and $\triangle+k \square$. We list our main results in the following theorems and corollaries.

Theorem 1.1 Let $a_{k}(n)$ and $b_{k}(n)$ be the number of representations of $n$ as $\triangle_{1}+2 k \triangle_{2}$ and $\triangle+k \square$, respectively. Then

$$
\begin{aligned}
\sum_{n=0}^{\infty} a_{k}((2 k+1) n) q^{n} & =\psi(q) \varphi\left(q^{k}\right)-q^{\frac{k(k+1)}{2}} \psi\left(q^{2 k+1}\right) \psi\left(q^{2 k(2 k+1)}\right) \\
\sum_{n=0}^{\infty} b_{1}(3 n+1) q^{n} & =4 \psi(q) \psi\left(q^{2}\right)-\psi\left(q^{3}\right) \varphi\left(q^{3}\right) \\
\sum_{n=0}^{\infty} b_{2}(5 n+3) q^{n} & =4 \psi(q) \psi\left(q^{4}\right)-\psi\left(q^{5}\right) \varphi\left(q^{10}\right) \\
\sum_{n=0}^{\infty} b_{3}(7 n+6) q^{n} & =4 \psi(q) \psi\left(q^{6}\right)-\psi\left(q^{7}\right) \varphi\left(q^{21}\right)
\end{aligned}
$$

where $k$ is a positive integer so that $2 k+1$ is odd prime.

Theorem 1.2 Let $a_{2}(n)$ and $b_{2}(n)$ be the number of representations of $n$ as $\triangle_{1}+4 \triangle_{2}$ and $\triangle+2 \square$, respectively. Then

$$
\begin{gathered}
a_{2}(625 n+390)-2 a_{2}(25 n+15)+a_{2}(n)=0, \\
b_{2}(625 n+78)-2 b_{2}(25 n+3)+b_{2}(n)=0 .
\end{gathered}
$$


The identities (1.5) and (1.6) are analogous to the Hirschhorn [2] identities

$$
\begin{gathered}
g(625 n+390)-6 g(25 n+15)+25 g(n)=0, \\
h(625 n+78)-6 h(25 n+3)+25 h(n)=0 .
\end{gathered}
$$

Corollary 1.1 Let $b_{k}(n)$ be the number of representations of $n$ as $\triangle+k \square$. Then for each $n, \alpha \geq 0$,

$$
\begin{aligned}
& \sum_{n=0}^{\infty} b_{1}\left(3^{2 \alpha} n+\frac{3^{2 \alpha}-1}{8}\right) q^{n} \equiv 3^{\alpha} \psi(q) \varphi(q) \quad\left(\bmod 2^{2}\right) \\
& \sum_{n=0}^{\infty} b_{2}\left(5^{2 \alpha} n+\frac{5^{2 \alpha}-1}{8}\right) q^{n} \equiv 3^{\alpha} \psi(q) \varphi\left(q^{2}\right) \quad\left(\bmod 2^{2}\right) \\
& \sum_{n=0}^{\infty} b_{3}\left(7^{2 \alpha} n+\frac{7^{2 \alpha}-1}{8}\right) q^{n} \equiv 3^{\alpha} \psi(q) \varphi\left(q^{3}\right) \quad\left(\bmod 2^{2}\right) .
\end{aligned}
$$

Corollary 1.2 Let $a_{2}(n)$ and $b_{2}(n)$ be the number of representations of $n$ as $\triangle_{1}+4 \triangle_{2}$ and $\triangle+2 \square$, respectively. Then for all $n, \alpha \geq 0$,

$$
\begin{aligned}
a_{2}\left(5^{4 \alpha} n+\frac{5\left(5^{4 \alpha}-1\right)}{8}\right) & \equiv a_{2}(n) \quad(\bmod 2), \\
b_{2}\left(5^{4 \alpha} n+\frac{5^{4 \alpha}-1}{8}\right) & \equiv b_{2}(n) \quad(\bmod 2) .
\end{aligned}
$$

\section{Preliminaries}

For $|a b|<1$, Ramanujan's general theta function $f(a, b)$ is defined by

$$
\begin{aligned}
f(a, b) & =\sum_{n=-\infty}^{\infty} a^{n(n+1) / 2} b^{n(n-1) / 2} \\
& =(-a ; a b)_{\infty}(-b ; a b)_{\infty}(a b ; a b)_{\infty} .
\end{aligned}
$$

Important special cases of $f(a, b)$ are

$$
\varphi(q):=f(q, q)=1+2 \sum_{n=1}^{\infty} q^{n^{2}}=\sum_{n=-\infty}^{\infty} q^{n^{2}}=\left(-q ; q^{2}\right)_{\infty}^{2}\left(q^{2} ; q^{2}\right)_{\infty}
$$

and

$$
\psi(q):=\frac{1}{2} f(1, q)=f\left(q, q^{3}\right)=\sum_{n=0}^{\infty} q^{\left(n^{2}+n\right) / 2}=\sum_{n=-\infty}^{\infty} q^{2 n^{2}+n}=\frac{\left(q^{2} ; q^{2}\right)_{\infty}}{\left(q ; q^{2}\right)_{\infty}} .
$$


From (2.3) and (2.4), we can see that

$$
\varphi(q)=\varphi\left(q^{4}\right)+2 q \psi\left(q^{8}\right)
$$

\section{Proofs of main results}

Proof of Theorem 1.1 Let us write

$$
E_{k}(q)=\psi(q) \psi\left(q^{2 k}\right)
$$

Using (2.4) in $E_{k}(q)$, we obtain

$$
E_{k}(q)=\sum_{m=-\infty}^{\infty} q^{2 m^{2}+m} \sum_{n=-\infty}^{\infty} q^{2 k\left(2 n^{2}+n\right)} .
$$

Replacing $q$ by $q^{8}$ and then multiplying both sides of (3.1) by $q^{2 k+1}$, we find that

$$
\begin{aligned}
q^{2 k+1} E_{k}\left(q^{8}\right) & =\sum_{m, n=-\infty}^{\infty} q^{(4 m+1)^{2}+2 k(4 n+1)^{2}} \\
& =\sum_{x, y \equiv 1(\bmod 4)} q^{x^{2}+2 k y^{2}} .
\end{aligned}
$$

If we write

$$
E_{k}(q)=E_{k}^{0}(q)+E_{k}^{1}(q)+\cdots+E_{k}^{2 k}(q),
$$

where $E_{k}^{i}(q)$ consists of all those terms in $E_{k}(q)$ in which powers of $q$ are congruent to $i$ modulo $2 k+1$, then from (3.2) and (3.3), we see that

$$
q^{2 k+1} E_{k}^{0}\left(q^{8}\right)=\sum_{x, y \equiv 1(\bmod 4)} q^{x^{2}+2 k y^{2}},
$$

where $x^{2}+2 k y^{2} \equiv 0(\bmod 2 k+1)$. The solution of $x^{2}+2 k y^{2} \equiv 0(\bmod 2 k+1)$ is $x \equiv \pm y(\bmod 2 k+1)$, and from (3.4) it follows that

$$
\begin{aligned}
q^{2 k+1} E_{k}^{0}\left(q^{8}\right)= & \sum_{\substack{x \equiv y \equiv 1(\bmod 4) \\
x \equiv y(\bmod 2 k+1)}} q^{x^{2}+2 k y^{2}}+\sum_{\substack{x \equiv y \equiv 1(\bmod 4) \\
x \equiv-y(\bmod 2 k+1)}} q^{x^{2}+2 k y^{2}} \\
& -\sum_{\substack{x \equiv y \equiv 1(\bmod 4) \\
x \equiv y \equiv 0(\bmod 2 k+1)}} q^{x^{2}+2 k y^{2}} .
\end{aligned}
$$

In the first sum of (3.5), we have $x \equiv y(\bmod 2 k+1), 4 m+1 \equiv 4 n+1(\bmod 2 k+1)$, $m-n \equiv 0(\bmod 2 k+1), m-n=(2 k+1) u, m+2 k n=(2 k+1) v, m=2 k u+v$, $n=v-u$, 


$$
x=8 k u+4 v+1, \text { and } y=4 v-4 u+1 .
$$

In the second sum of $(3.5), x \equiv-y(\bmod 2 k+1), 4 m+1 \equiv-4 n-1(\bmod 2 k+1)$, $4(m+n) \equiv-2(\bmod 2 k+1), 4(m+n) \equiv-2+2(2 k+1)(\bmod 2 k+1), m+n \equiv$ $k(\bmod 2 k+1), m+n=(2 k+1) v+k, m-2 k n=(2 k+1) u+k, m=u+2 k v+k$, $n=v-u$,

$$
x=4 u+8 k v+4 k+1, \text { and } y=4 v-4 u+1 .
$$

In the third sum of (3.5),

$$
x=4(2 k+1) u+2 k+1 \text { and } y=4(2 k+1) v+2 k+1 \text {. }
$$

Employing (3.6), (3.7), and (3.8) in (3.5), we obtain

$$
\begin{aligned}
q^{2 k+1} E_{k}^{0}\left(q^{8}\right)= & \sum_{u, v=-\infty}^{\infty} q^{(8 k u+4 v+1)^{2}+2 k(4 v-4 u+1)^{2}} \\
& +\sum_{u, v=-\infty}^{\infty} q^{(4 u+8 k v+4 k+1)^{2}+2 k(4 v-4 u+1)^{2}} \\
& -\sum_{u, v=-\infty}^{\infty} q^{((4 u+1)(2 k+1))^{2}+2 k((4 v+1)(2 k+1))^{2}} \\
= & \sum_{u, v=-\infty}^{\infty} q^{16(2 k+1) v^{2}+8(2 k+1) v+16\left(4 k^{2}+2 k\right) u^{2}+2 k+1} \\
& +\sum_{u, v=-\infty}^{\infty} q^{16(2 k+1) u^{2}+8(2 k+1) u+16\left(4 k^{2}+2 k\right) v^{2}+16\left(4 k^{2}+2 k\right) v+16 k^{2}+10 k+1} \\
& -\sum_{u, v=-\infty}^{\infty} q^{16(2 k+1)^{2} u^{2}+8(2 k+1)^{2} u+32 k(2 k+1)^{2} v^{2}+16 k(2 k+1)^{2} v+(2 k+1)^{3}} .
\end{aligned}
$$

On simplification, the above equation reduces to

$$
\begin{aligned}
E_{k}^{0}(q)= & \sum_{v=-\infty}^{\infty} q^{(2 k+1)\left(2 v^{2}+v\right)} \sum_{u=-\infty}^{\infty} q^{4 k(2 k+1) u^{2}} \\
& +q^{k(2 k+1)} \sum_{u=-\infty}^{\infty} q^{(2 k+1)\left(2 u^{2}+u\right)} \sum_{v=-\infty}^{\infty} q^{4 k(2 k+1)\left(v^{2}+v\right)} \\
& -q^{\frac{k(k+1)(2 k+1)}{2}} \sum_{u=-\infty}^{\infty} q^{(2 k+1)^{2}\left(2 u^{2}+u\right)} \sum_{v=-\infty}^{\infty} q^{2 k(2 k+1)^{2}\left(2 v^{2}+v\right)}
\end{aligned}
$$


From (2.3), (2.4), and (3.9), we have

$$
\begin{aligned}
E_{k}^{0}(q)= & \psi\left(q^{2 k+1}\right)\left(\varphi\left(q^{4 k(2 k+1)}\right)+2 q^{k(2 k+1)} \psi\left(q^{8 k(2 k+1)}\right)\right) \\
& -q^{\frac{k(k+1)(2 k+1)}{2}} \psi\left(q^{(2 k+1)^{2}}\right) \psi\left(q^{2 k(2 k+1)^{2}}\right) .
\end{aligned}
$$

From (2.5) and (3.10), we find that

$$
E_{k}^{0}(q)=\psi\left(q^{2 k+1}\right) \varphi\left(q^{k(2 k+1)}\right)-q^{\frac{k(k+1)(2 k+1)}{2}} \psi\left(q^{(2 k+1)^{2}}\right) \psi\left(q^{2 k(2 k+1)^{2}}\right) .
$$

The identity (3.11) can be written as

$$
\begin{aligned}
\sum_{n=0}^{\infty} a_{k}((2 k+1) n) q^{(2 k+1) n}= & \psi\left(q^{2 k+1}\right) \varphi\left(q^{k(2 k+1)}\right) \\
& -q^{\frac{k(k+1)(2 k+1)}{2}} \psi\left(q^{(2 k+1)^{2}}\right) \psi\left(q^{2 k(2 k+1)^{2}}\right) .
\end{aligned}
$$

Now replacing $q^{2 k+1}$ by $q$ in (3.12), we arrive at (1.1).

Let us write $F_{2}(q)=\psi(q) \varphi(q)$, and using (2.5), we find that

$$
\begin{aligned}
F_{2}(q) & =\psi(q)\left(\varphi\left(q^{4}\right)+2 q \psi\left(q^{8}\right)\right) \\
& =\sum_{m, n=-\infty}^{\infty} q^{2 m^{2}+m+4 n^{2}}+q \sum_{m, n=-\infty}^{\infty} q^{2 m^{2}+m+4\left(n^{2}+n\right)}
\end{aligned}
$$

Replacing $q$ by $q^{8}$ and then multiplying both sides of (3.13) by $q$, we obtain

$$
\begin{aligned}
q F_{2}\left(q^{8}\right) & =\sum_{m, n=-\infty}^{\infty} q^{(4 m+1)^{2}+2(4 n)^{2}}+\sum_{m, n=-\infty}^{\infty} q^{(4 m+1)^{2}+2(4 n+2)^{2}} \\
& =\sum_{x \equiv 1, y \equiv 0(\bmod 4)} q^{x^{2}+2 y^{2}}+\sum_{x \equiv 1, y \equiv 2(\bmod 4)} q^{x^{2}+2 y^{2}} .
\end{aligned}
$$

If we write

$$
F_{2}(q)=F_{2}^{0}(q)+F_{2}^{1}(q)+F_{2}^{2}(q)
$$

where $F_{2}^{i}(q)$ consists of all those terms in $F_{2}(q)$ in which powers of $q$ are congruent to $i$ modulo 3 , then from (3.14) and (3.15), we see that

$$
q F_{2}^{1}\left(q^{8}\right)=\sum_{x \equiv 1, y \equiv 0(\bmod 4)} q^{x^{2}+2 y^{2}}+\sum_{x \equiv 1, y \equiv 2(\bmod 4)} q^{x^{2}+2 y^{2}}
$$


where, in both sums, $x^{2}+2 y^{2} \equiv 0(\bmod 3)$. The solution of $x^{2}+2 y^{2} \equiv 0(\bmod 3)$ is $x \equiv \pm y(\bmod 3)$, and from $(3.16)$ it follows that

$$
\begin{aligned}
& q F_{2}^{1}\left(q^{8}\right) \\
& =\sum_{\substack{x \equiv 1, y \equiv 0(\bmod 4) \\
x \equiv y(\bmod 3)}} q^{x^{2}+2 y^{2}}+\sum_{\substack{x \equiv 1, y \equiv 0(\bmod 4) \\
x \equiv-y(\bmod 3)}} q^{x^{2}+2 y^{2}}-\sum_{\substack{x \equiv 1, y \equiv 0(\bmod 4) \\
x \equiv y \equiv 0(\bmod 3)}} q^{x^{2}+2 y^{2}} \\
& \quad+\sum_{\substack{x \equiv 1, y \equiv 2(\bmod 4) \\
x \equiv y(\bmod 3)}} q^{x^{2}+2 y^{2}}+\sum_{\substack{x \equiv 1, y \equiv 2(\bmod 4) \\
x \equiv-y(\bmod 3)}} q^{x^{2}+2 y^{2}}-\sum_{\substack{x \equiv 1, y \equiv 2(\bmod 4) \\
x \equiv y \equiv 0(\bmod 3)}} q^{x^{2}+2 y^{2}} .
\end{aligned}
$$

In the first sum of (3.17), we have $4 m+1 \equiv 4 n(\bmod 3), 4(m-n) \equiv-1(\bmod 3)$, $4(m-n) \equiv-1+9(\bmod 3), m-n \equiv 2(\bmod 3), m-n \equiv-1(\bmod 3), m+2 n \equiv$ $-1(\bmod 3), m-n=-3 u-1, m+2 n=-3 v-1, m=-v-2 u-1, n=u-v$,

$$
x=-4 v-8 u-3, \text { and } y=4 u-4 v .
$$

In the second sum of (3.17), $4 m+1 \equiv-4 n(\bmod 3), 4(m+n) \equiv-1(\bmod 3)$, $4(m+n) \equiv-1+9(\bmod 3), m+n \equiv 2(\bmod 3), m+n \equiv-1(\bmod 3), m-2 n \equiv$ $-1(\bmod 3), m+n=-3 u-1, m-2 n=-3 v-1, m=-v-2 u-1, n=v-u$,

$$
x=-4 v-8 u-3, \text { and } y=4 v-4 u .
$$

In the third sum of (3.17),

$$
x=12 u+3 \text { and } y=12 v .
$$

In the fourth sum of $(3.17), 4 m+1 \equiv 4 n+2(\bmod 3), 4(m-n) \equiv 1(\bmod 3)$, $m-n \equiv 1(\bmod 3), m+2 n \equiv-2(\bmod 3), m-n=3 u+1, m+2 n=-3 v-2$, $m=2 u-v, n=-u-v-1$,

$$
x=8 u-4 v+1, \text { and } y=-4 u-4 v-2 .
$$

In the fifth sum of $(3.17), 4 m+1 \equiv-4 n-2(\bmod 3), 4(m+n) \equiv-3(\bmod 3)$, $m+n \equiv 0(\bmod 3), m-2 n \equiv 0(\bmod 3), m+n=3 u, m-2 n=-3 v, m=2 u-v$, $n=u+v$,

$$
x=8 u-4 v+1 \text {, and } y=4 u+4 v+2 .
$$

In the sixth sum of (3.17),

$$
x=12 u+3 \text { and } y=12 v+6 .
$$


Invoking (3.18), (3.19), (3.20), (3.21), (3.22), and (3.23) in (3.17), we arrive at

$$
\begin{aligned}
q F_{2}^{1}\left(q^{8}\right)= & \sum_{u, v=-\infty}^{\infty} q^{(-4 v-8 u-3)^{2}+2(4 u-4 v)^{2}}+\sum_{u, v=-\infty}^{\infty} q^{(-4 v-8 u-3)^{2}+2(4 v-4 u)^{2}} \\
& -\sum_{u, v=-\infty}^{\infty} q^{(12 u+3)^{2}+2(12 v)^{2}}+\sum_{u, v=-\infty}^{\infty} q^{(8 u-4 v+1)^{2}+2(-4 u-4 v-2)^{2}} \\
& +\sum_{u, v=-\infty}^{\infty} q^{(8 u-4 v+1)^{2}+2(4 u+4 v+2)^{2}}-\sum_{u, v=-\infty}^{\infty} q^{(12 u+3)^{2}+2(12 v+6)^{2}} \\
= & \sum_{u, v=-\infty}^{\infty} q^{48\left(2 u^{2}+u\right)+24\left(2 v^{2}+v\right)+9}+\sum_{u, v=-\infty}^{\infty} q^{48\left(2 u^{2}+u\right)+24\left(2 v^{2}+v\right)+9} \\
& -\sum_{u, v=-\infty}^{\infty} q^{72\left(2 u^{2}+u\right)+288 v^{2}+9}+\sum_{u, v=-\infty}^{\infty} q^{48\left(2 u^{2}+u\right)+24\left(2 v^{2}+v\right)+9} \\
& +\sum_{u, v=-\infty}^{\infty} q^{48\left(2 u^{2}+u\right)+24\left(2 v^{2}+v\right)+9}-\sum_{u, v=-\infty}^{\infty} q^{72\left(2 u^{2}+u\right)+288\left(v^{2}+v\right)+81} .
\end{aligned}
$$

Dividing throughout by $q$, replacing $q^{8}$ by $q$, and separating the terms of $u$ and $v$ in the above equation, we obtain

$$
\begin{aligned}
F_{2}^{1}(q)= & 4 q \sum_{u=-\infty}^{\infty} q^{12 u^{2}+6 u} \sum_{v=-\infty}^{\infty} q^{6 v^{2}+3 v}-q \sum_{u=-\infty}^{\infty} q^{18 u^{2}+9 u} \sum_{v=-\infty}^{\infty} q^{36 v^{2}} \\
& -q^{10} \sum_{u=-\infty}^{\infty} q^{18 u^{2}+9 u} \sum_{v=-\infty}^{\infty} q^{36 v^{2}+36 v} \\
= & 4 q \psi\left(q^{6}\right) \psi\left(q^{3}\right)-q \psi\left(q^{9}\right) \varphi\left(q^{36}\right)-2 q^{10} \psi\left(q^{9}\right) \psi\left(q^{72}\right) \\
= & 4 q \psi\left(q^{6}\right) \psi\left(q^{3}\right)-q \psi\left(q^{9}\right)\left(\varphi\left(q^{36}\right)+2 q^{9} \psi\left(q^{72}\right)\right) \\
= & 4 q \psi\left(q^{6}\right) \psi\left(q^{3}\right)-q \psi\left(q^{9}\right) \varphi\left(q^{9}\right) .
\end{aligned}
$$

The above identity can be written as

$$
\sum_{n=0}^{\infty} b_{1}(3 n+1) q^{3 n+1}=4 q \psi\left(q^{6}\right) \psi\left(q^{3}\right)-q \psi\left(q^{9}\right) \varphi\left(q^{9}\right) .
$$

Dividing throughout by $q$ and then replacing $q^{3}$ by $q$ in (3.24), we obtain (1.2). 
Let $F_{4}(q)=\psi(q) \varphi\left(q^{2}\right)$, and using (2.5), we find that

$$
\begin{aligned}
F_{4}(q) & =\psi(q)\left(\varphi\left(q^{8}\right)+2 q^{2} \psi\left(q^{16}\right)\right) \\
& =\sum_{m, n=-\infty}^{\infty} q^{2 m^{2}+m+8 n^{2}}+q^{2} \sum_{m, n=-\infty}^{\infty} q^{2 m^{2}+m+8\left(n^{2}+n\right)}
\end{aligned}
$$

Replacing $q$ by $q^{8}$ and then multiplying throughout by $q$ in the above equation, we obtain

$$
\begin{aligned}
q F_{4}\left(q^{8}\right) & =\sum_{m, n=-\infty}^{\infty} q^{(4 m+1)^{2}+4(4 n)^{2}}+\sum_{m, n=-\infty}^{\infty} q^{(4 m+1)^{2}+4(4 n+2)^{2}} \\
& =\sum_{x \equiv 1, y \equiv 0(\bmod 4)} q^{x^{2}+4 y^{2}}+\sum_{x \equiv 1, y \equiv 2(\bmod 4)} q^{x^{2}+4 y^{2}} .
\end{aligned}
$$

Let us write

$$
F_{4}(q)=F_{4}^{0}(q)+F_{4}^{1}(q)+F_{4}^{2}(q)+F_{4}^{3}(q)+F_{4}^{4}(q)
$$

where $F_{4}^{i}(q)$ consists of all those terms in $F_{4}(q)$ in which powers of $q$ are congruent to $i$ modulo 5. From (3.26) and (3.27), we see that

$$
q F_{4}^{3}\left(q^{8}\right)=\sum_{x \equiv 1, y \equiv 0(\bmod 4)} q^{x^{2}+4 y^{2}}+\sum_{x \equiv 1, y \equiv 2(\bmod 4)} q^{x^{2}+4 y^{2}}
$$

where, in both sums, $x^{2}+4 y^{2} \equiv 0(\bmod 5)$. The solution of $x^{2}+4 y^{2} \equiv 0(\bmod 5)$ is $x \equiv \pm y(\bmod 5)$, and it follows that

$$
\begin{aligned}
& q F_{4}^{3}\left(q^{8}\right) \\
& =\sum_{\substack{x \equiv 1, y \equiv 0(\bmod 4) \\
x \equiv y(\bmod 5)}} q^{x^{2}+4 y^{2}}+\sum_{\substack{x \equiv 1, y \equiv 0(\bmod 4) \\
x \equiv-y(\bmod 5)}} q^{x^{2}+4 y^{2}}-\sum_{\substack{x \equiv 1, y \equiv 0(\bmod 4) \\
x \equiv y \equiv 0(\bmod 5)}} q^{x^{2}+4 y^{2}} \\
& \quad+\sum_{\substack{x \equiv 1, y \equiv 2(\bmod 4) \\
x \equiv y(\bmod 5)}} q^{x^{2}+4 y^{2}}+\sum_{\substack{x \equiv 1, y \equiv 2(\bmod 4) \\
x \equiv-y(\bmod 5)}} q^{x^{2}+4 y^{2}}-\sum_{\substack{x \equiv 1, y \equiv 2(\bmod 4) \\
x \equiv y \equiv 0(\bmod 5)}} q^{x^{2}+4 y^{2}} .
\end{aligned}
$$

In the first sum of (3.29), we have $x \equiv y(\bmod 5), 4 m+1 \equiv 4 n(\bmod 5), 4(m-n) \equiv$ $-1(\bmod 5), m-n \equiv 1(\bmod 5), m+4 n \equiv 1(\bmod 5), m-n=5 u+1, m+4 n=5 v+1$, $m=4 u+v+1, n=v-u$,

$$
x=16 u+4 v+5 \text {, and } y=4 v-4 u .
$$


In the second sum of (3.29), $4 m+1 \equiv-4 n(\bmod 5), 4(m+n) \equiv-1(\bmod 5)$, $4(m+n) \equiv-1+5(\bmod 5), m+n \equiv 1(\bmod 5), m-4 n \equiv 1(\bmod 5), m+n=5 u+1$, $m-4 n=5 v+1, m=4 u+v+1, n=u-v$,

$$
x=16 u+4 v+5, \text { and } y=4 u-4 v .
$$

In the third sum of (3.29),

$$
x=20 u+5 \text { and } y=20 v .
$$

In the fourth sum of (3.29), $4 m+1 \equiv 4 n+2(\bmod 5), 4(m-n) \equiv 1(\bmod 5)$, $m-n \equiv-1(\bmod 5), m+4 n \equiv-1(\bmod 5), m-n=-5 u-1, m+4 n=5 v-1$, $m=v-4 u-1, n=u+v$,

$$
x=4 v-16 u-3, \text { and } y=4 u+4 v+2 .
$$

In the fifth sum of (3.29), $4 m+1 \equiv-4 n-2(\bmod 5), 4(m+n) \equiv-3(\bmod 5)$, $m+n \equiv-2(\bmod 5), m-4 n \equiv 3(\bmod 5), m+n=-5 u-2, m-4 n=5 v+3$, $m=v-4 u-1, n=-v-u-1$,

$$
x=4 v-16 u-3, \text { and } y=-4 u-4 v-2 .
$$

In the sixth sum of (3.29),

$$
x=20 u+5 \text { and } y=20 v+10 .
$$

Employing (3.30), (3.31), (3.32), (3.33), (3.34), and (3.35) in (3.29), we obtain

$$
\begin{aligned}
q F_{4}^{3}\left(q^{8}\right)= & \sum_{u, v=-\infty}^{\infty} q^{(16 u+4 v+5)^{2}+4(4 v-4 u)^{2}}+\sum_{u, v=-\infty}^{\infty} q^{(16 u+4 v+5)^{2}+4(4 u-4 v)^{2}} \\
& -\sum_{u, v=-\infty}^{\infty} q^{(20 u+5)^{2}+4(20 v)^{2}}+\sum_{u, v=-\infty}^{\infty} q^{(4 v-16 u-3)^{2}+4(4 v+4 u+2)^{2}} \\
& +\sum_{u, v=-\infty}^{\infty} q^{(4 v-16 u-3)^{2}+4(-4 v-4 u-2)^{2}}-\sum_{u, v=-\infty}^{\infty} q^{(20 u+5)^{2}+4(20 v+10)^{2}} \\
= & \sum_{u, v=-\infty}^{\infty} q^{40\left(2 v^{2}+v\right)+160\left(2 u^{2}+u\right)+25}+\sum_{u, v=-\infty}^{\infty} q^{40\left(2 v^{2}+v\right)+160\left(2 u^{2}+u\right)+25} \\
& -\sum_{u, v=-\infty}^{\infty} q^{200\left(2 u^{2}+u\right)+1600 v^{2}+25}+\sum_{u, v=-\infty}^{\infty} q^{40\left(2 v^{2}+v\right)+160\left(2 u^{2}+u\right)+25} \\
& +\sum_{u, v=-\infty}^{\infty} q^{40\left(2 v^{2}+v\right)+160\left(2 u^{2}+u\right)+25}-\sum_{u, v=-\infty}^{\infty} q^{200\left(2 u^{2}+u\right)+1600\left(v^{2}+v\right)+425}
\end{aligned}
$$


Dividing throughout by $q$, replacing $q^{8}$ by $q$, and then separating the terms containing $u$ and $v$ in the above equation, we find that

$$
\begin{aligned}
F_{4}^{3}(q)= & 4 q^{3} \sum_{v=-\infty}^{\infty} q^{10 v^{2}+5 v} \sum_{u=-\infty}^{\infty} q^{40 u^{2}+20 u}-q^{3} \sum_{u=-\infty}^{\infty} q^{50 u^{2}+25 u} \sum_{v=-\infty}^{\infty} q^{200 v^{2}} \\
& -q^{53} \sum_{u=-\infty}^{\infty} q^{50 u^{2}+25 u} \sum_{v=-\infty}^{\infty} q^{200 v^{2}+200 v}
\end{aligned}
$$

Using (2.3) and (2.4) in (3.36), we obtain

$$
F_{4}^{3}(q)=4 q^{3} \psi\left(q^{5}\right) \psi\left(q^{20}\right)-q^{3} \psi\left(q^{25}\right)\left(\varphi\left(q^{200}\right)+2 q^{50} \psi\left(q^{400}\right)\right)
$$

From (2.5) and (3.37), we see that

$$
F_{4}^{3}(q)=4 q^{3} \psi\left(q^{5}\right) \psi\left(q^{20}\right)-q^{3} \psi\left(q^{25}\right) \varphi\left(q^{50}\right) .
$$

From (3.38), it follows that

$$
\sum_{n=0}^{\infty} b_{2}(5 n+3) q^{5 n+3}=4 q^{3} \psi\left(q^{5}\right) \psi\left(q^{20}\right)-q^{3} \psi\left(q^{25}\right) \varphi\left(q^{50}\right)
$$

Dividing throughout by $q^{3}$ and then replacing $q^{5}$ by $q$ in (3.39), we arrive at (1.3).

Let $F_{6}(q)=\psi(q) \varphi\left(q^{3}\right)$, and using (2.5), we find that

$$
\begin{aligned}
F_{6}(q) & =\psi(q)\left(\varphi\left(q^{12}\right)+2 q^{3} \psi\left(q^{24}\right)\right) \\
& =\sum_{m, n=-\infty}^{\infty} q^{2 m^{2}+m+12 n^{2}}+q \sum_{m, n=-\infty}^{\infty} q^{2 m^{2}+m+12\left(n^{2}+n\right)}
\end{aligned}
$$

Replacing $q$ by $q^{8}$ and then multiplying both sides of (3.40) by $q$, we see that

$$
\begin{aligned}
q F_{6}\left(q^{8}\right) & =\sum_{m, n=-\infty}^{\infty} q^{(4 m+1)^{2}+6(4 n)^{2}}+\sum_{m, n=-\infty}^{\infty} q^{(4 m+1)^{2}+6(4 n+2)^{2}} \\
& =\sum_{x \equiv 1, y \equiv 0(\bmod 4)} q^{x^{2}+6 y^{2}}+\sum_{x \equiv 1, y \equiv 2(\bmod 4)} q^{x^{2}+6 y^{2}} .
\end{aligned}
$$

If we write

$$
F_{6}(q)=F_{6}^{0}(q)+F_{6}^{1}(q)+\cdots+F_{6}^{6}(q),
$$


where $F_{6}^{i}(q)$ consists of all those terms in $F_{6}(q)$ in which powers of $q$ are congruent to $i$ modulo 7, then from (3.41) and (3.42), we find that

$$
q F_{6}^{6}\left(q^{8}\right)=\sum_{x \equiv 1, y \equiv 0(\bmod 4)} q^{x^{2}+6 y^{2}}+\sum_{x \equiv 1, y \equiv 2(\bmod 4)} q^{x^{2}+6 y^{2}}
$$

where, in both sums, $x^{2}+6 y^{2} \equiv 0(\bmod 7)$. The solution of $x^{2}+6 y^{2} \equiv 0(\bmod 7)$ is $x \equiv \pm y(\bmod 7)$, and from $(3.43)$, it follows that

$$
\begin{aligned}
& q F_{6}^{6}\left(q^{8}\right) \\
& =\sum_{\substack{x \equiv 1, y \equiv 0(\bmod 4) \\
x \equiv y(\bmod 7)}} q^{x^{2}+6 y^{2}}+\sum_{\substack{x \equiv 1, y \equiv 0(\bmod 4) \\
x \equiv-y(\bmod 7)}} q^{x^{2}+6 y^{2}}-\sum_{\substack{x \equiv 1, y \equiv 0(\bmod 4) \\
x \equiv y \equiv 0(\bmod 7)}} q^{x^{2}+6 y^{2}} \\
& +\sum_{\substack{x \equiv 1, y \equiv 2(\bmod 4) \\
x \equiv y(\bmod 7)}} q^{x^{2}+6 y^{2}}+\sum_{\substack{x \equiv 1, y \equiv 2(\bmod 4) \\
x \equiv-y(\bmod 7)}} q^{x^{2}+6 y^{2}}-\sum_{\substack{x \equiv 1, y \equiv 2(\bmod 4) \\
x \equiv y \equiv 0(\bmod 7)}} q^{x^{2}+6 y^{2}} .
\end{aligned}
$$

In the first sum of (3.44), we have $4 m+1 \equiv 4 n(\bmod 7), 4(m-n) \equiv-1(\bmod 7)$, $4(m-n) \equiv-8(\bmod 7), m-n \equiv-2(\bmod 7), m+6 n \equiv-2(\bmod 7), m-n=-7 v-2$, $m+6 n=-7 u-2, m=-u-6 v-2, n=v-u$,

$$
x=-4 u-24 v-7, \text { and } y=4 v-4 u .
$$

In the second sum of $(3.44), 4 m+1 \equiv-4 n(\bmod 7), 4(m+n) \equiv-1(\bmod 7)$, $4(m+n) \equiv-8(\bmod 7), m+n \equiv-2(\bmod 7), m-6 n \equiv-2(\bmod 7), m+n=-7 v-2$, $m-6 n=-7 u-2, m=-6 v-u-2, n=u-v$,

$$
x=-24 v-4 u-7, \text { and } y=4 u-4 v .
$$

In the third sum of (3.44),

$$
x=28 u+7 \text { and } y=28 v .
$$

In the fourth sum of $(3.44), 4 m+1 \equiv 4 n+2(\bmod 7), 4(m-n) \equiv 1(\bmod 7)$, $m-n \equiv 2(\bmod 7), m+6 n \equiv-5(\bmod 7), m-n=7 v+2, m+6 n=-7 u-5$, $m=6 v-u+1, n=-v-u-1$,

$$
x=24 v-4 u+5 \text {, and } y=-4 u-4 v-2 .
$$

In the fifth sum of $(3.44), 4 m+1 \equiv-4 n-2(\bmod 7), 4(m+n) \equiv-3(\bmod 7)$, $m+n \equiv 1(\bmod 7), m-6 n \equiv 1(\bmod 7), m+n=7 v+1, m-6 n=-7 u+1$, $m=6 v-u+1, n=u+v$,

$$
x=24 v-4 u+5, \text { and } y=4 u+4 v+2 .
$$


In the sixth sum of (3.44),

$$
x=28 u+7 \text { and } y=28 v+14 .
$$

Invoking (3.45), (3.46), (3.47), (3.48), (3.49), and (3.50) in (3.44), we find that

$$
\begin{aligned}
q F_{6}^{6}\left(q^{8}\right)= & \sum_{u, v=-\infty}^{\infty} q^{(-4 u-24 v-7)^{2}+6(4 v-4 u)^{2}}+\sum_{u, v=-\infty}^{\infty} q^{(-24 v-4 u-7)^{2}+6(4 u-4 v)^{2}} \\
& -\sum_{u, v=-\infty}^{\infty} q^{(28 u+7)^{2}+6(28 v)^{2}}+\sum_{u, v=-\infty}^{\infty} q^{(24 v-4 u+5)^{2}+6(-4 u-4 v-2)^{2}} \\
& +\sum_{u, v=-\infty}^{\infty} q^{(24 v-4 u+5)^{2}+6(4 u+4 v+2)^{2}}-\sum_{u, v=-\infty}^{\infty} q^{(28 u+7)^{2}+6(28 v+14)^{2}}
\end{aligned}
$$

After simplification of (3.51), we obtain

$$
\begin{aligned}
q F_{6}^{6}\left(q^{8}\right)= & \sum_{u, v=-\infty}^{\infty} q^{56\left(2 u^{2}+u\right)+336\left(2 v^{2}+v\right)+49}+\sum_{u, v=-\infty}^{\infty} q^{56\left(2 u^{2}+u\right)+336\left(2 v^{2}+v\right)+49} \\
& -\sum_{u, v=-\infty}^{\infty} q^{392\left(2 u^{2}+u\right)+4704 v^{2}+49}+\sum_{u, v=-\infty}^{\infty} q^{56\left(2 u^{2}+u\right)+336\left(2 v^{2}+v\right)+49} \\
& +\sum_{u, v=-\infty}^{\infty} q^{56\left(2 u^{2}+u\right)+336\left(2 v^{2}+v\right)+49}-\sum_{u, v=-\infty}^{\infty} q^{392\left(2 u^{2}+u\right)+4704\left(v^{2}+v\right)+1225}
\end{aligned}
$$

Dividing throughout by $q$, replacing $q^{8}$ by $q$, and separating the terms of $u$ and $v$ in (3.52), we obtain

$$
\begin{aligned}
F_{6}^{6}(q)= & 4 q^{6} \sum_{u=-\infty}^{\infty} q^{14 u^{2}+7 u} \sum_{v=-\infty}^{\infty} q^{84 v^{2}+42 v}-q^{6} \sum_{u=-\infty}^{\infty} q^{98 u^{2}+49 u} \sum_{v=-\infty}^{\infty} q^{588 v^{2}} \\
& -q^{153} \sum_{u=-\infty}^{\infty} q^{98 u^{2}+49 u} \sum_{v=-\infty}^{\infty} q^{588 v^{2}+588 v}
\end{aligned}
$$

From (2.3), (2.4), and (3.53), we see that

$$
F_{6}^{6}(q)=4 q^{6} \psi\left(q^{7}\right) \psi\left(q^{42}\right)-q^{6} \psi\left(q^{49}\right)\left(\varphi\left(q^{588}\right)+2 q^{147} \psi\left(q^{1176}\right)\right)
$$

From (2.5) and (3.54), we obtain

$$
F_{6}^{6}(q)=4 q^{6} \psi\left(q^{7}\right) \psi\left(q^{42}\right)-q^{6} \psi\left(q^{49}\right) \varphi\left(q^{147}\right) .
$$


The above equation can be written as

$$
\sum_{n=0}^{\infty} b_{3}(7 n+6) q^{7 n+6}=4 q^{6} \psi\left(q^{7}\right) \psi\left(q^{42}\right)-q^{6} \psi\left(q^{49}\right) \varphi\left(q^{147}\right) .
$$

Dividing throughout by $q^{6}$ and then replacing $q^{7}$ by $q$ in (3.56), we arrive at (1.4). This completes the proof.

Proof of Theorem 1.2 Put $k=2$ in (1.1), we find that

$$
\sum_{n=0}^{\infty} a_{2}(5 n) q^{n}=\sum_{n=0}^{\infty} b_{2}(n) q^{n}-\sum_{n=0}^{\infty} a_{2}(n) q^{5 n+3}
$$

which yields

$$
a_{2}(n)+a_{2}(25 n+15)=b_{2}(5 n+3) .
$$

From (1.3), it follows that

$$
\sum_{n=0}^{\infty} b_{2}(5 n+3) q^{n}=4 \sum_{n=0}^{\infty} a_{2}(n) q^{n}-\sum_{n=0}^{\infty} b_{2}(n) q^{5 n}
$$

Equating coefficients of $q^{5 n}$ in (3.59), we obtain

$$
b_{2}(n)+b_{2}(25 n+3)=4 a_{2}(5 n) .
$$

Equations (1.5) and (1.6) follow from (3.58) and (3.60). This completes the proof.

Proof of Corollary 1.1 From Eq. (1.2), we see that

$$
\sum_{n=0}^{\infty} b_{1}(3 n+1) q^{n} \equiv 3 \psi\left(q^{3}\right) \varphi\left(q^{3}\right) \quad(\bmod 4)
$$

Equating coefficients of $q^{3 n}$ on both sides of (3.61), we find that

$$
\sum_{n=0}^{\infty} b_{1}(9 n+1) q^{n} \equiv 3 \psi(q) \varphi(q) \quad(\bmod 4)
$$

From (3.62) and by mathematical induction, we arrive at (1.7). Similarly, we obtain (1.8) and (1.9) from (1.3) and (1.4), respectively. This completes the proof.

Proof of Corollary 1.2 In view of Eq. (1.5), we see that

$$
a_{2}(625 n+390) \equiv a_{2}(n)(\bmod 2)
$$

By mathematical induction on $n$ in (3.63), we arrive at (1.10). Similarly, we obtain (1.11) from (1.6). This completes the proof. 
Acknowledgments The authors would like to thank the anonymous referee for helpful suggestions and comments which greatly improved the original version of the manuscript.

\section{References}

1. Berndt, B.C.: Ramanujan's Notebooks, Part III. Springer, New York (1991)

2. Hirschhorn, M.D.: Some interesting q-series identities. Ramanujan J. 36, 297-304 (2015)

3. Hirschhorn, M.D., Sellers, J.A.: Infinitely many congruences modulo 5 for 4-colored Frobenius partitions. Ramanujan J. (2014). doi:10.1007/s11139-014-9652-x 